\title{
Interferometric characterization of few-mode fibers (FMF) for mode-division multiplexing (MDM)
}

Muliar, Olena; Usuga Castaneda, Mario A.; Rottwitt, Karsten; Lægsgaard, Jesper

\section{Published in:}

Proceedings of SPIE

Link to article, DOI:

$10.1117 / 12.2079413$

Publication date:

2015

Document Version

Publisher's PDF, also known as Version of record

Link back to DTU Orbit

Citation (APA):

Muliar, O., Usuga Castaneda, M. A., Rottwitt, K., \& Lægsgaard, J. (2015). Interferometric characterization of few-mode fibers (FMF) for mode-division multiplexing (MDM). In Y. G. Soskind, \& C. Olson (Eds.), Proceedings of SPIE (Vol. 9369). [936909] SPIE - International Society for Optical Engineering. Proceedings of SPIE - The International Society for Optical Engineering https://doi.org/10.1117/12.2079413

\section{General rights}

Copyright and moral rights for the publications made accessible in the public portal are retained by the authors and/or other copyright owners and it is a condition of accessing publications that users recognise and abide by the legal requirements associated with these rights.

- Users may download and print one copy of any publication from the public portal for the purpose of private study or research.

- You may not further distribute the material or use it for any profit-making activity or commercial gain

- You may freely distribute the URL identifying the publication in the public portal 


\title{
Interferometric characterization of few mode fibers (FMF) for mode division multiplexing (MDM)
}

\author{
O.Muliar*, M.A.Usuga, K.Rottwitt, J.Lægsgaard \\ Dept. of Photonics Engineering, Technical University of Denmark, Oersteds Plads, \\ 2800 Kgs. Lyngby, Denmark
}

\begin{abstract}
The rapid growth of global data traffic demands the continuous search for new technologies and systems that could increase transmission capacity in optical links and recent experiments show that to do so, it is advantageous to explore new degrees of freedom such as polarization, wavelength or optical modes.

Mode division multiplexing (MDM) appears in this context as a promising and viable solution for such capacity increase, since it utilizes multiple spatial modes of an optical fiber as individual communication channels for data transmission. In order to evaluate its performance, a MDM system requires advanced characterization methods with regard to the modal content of its photonics components and in particular of the fibers involved for data transmission.

In this contribution we present a time-domain interferometric technique for a full modal characterization of few mode fibers (FMF), commonly used in a MDM scenario. This experimental technique requires the use of a Mach-Zehnder interferometer, where the reference's path length is controlled by an optical delay line. The interference between the output beams of reference and fiber under test (FUT) is recorded on a CCD camera and a careful evaluation of the resulting interferograms allows us to have full access to key parameters such as number of modes, modal weight, differential time delay between propagating modes and intensity profiles.

In this work, we apply this simple and complete characterization method to the case of a short link with two optical modes propagating in a FMF, which illustrates its potential as a diagnostic tool for MDM systems.
\end{abstract}

Keywords: mode division multiplexing, few mode fiber, low coherence interferometry.

\section{INTRODUCTION}

Over the last few decades, optical communication technologies were tremendously developed and permitted to increase the transmitted data capacity in optical links beyond the $\mathrm{Tb} / \mathrm{s}$. To achieve such an improvement in transmission capacity using only single mode fibers, different techniques such as time-division multiplexing (TDM), wavelength-division multiplexing (WDM) and polarization-division multiplexing (PDM) were implemented in modern communication systems. However, the demand for transmission of even larger amounts of data continues to grow and requires the development of new optical communication technologies to overcome the capacity limit that is about to be reached in the near future. One of the possible solutions is mode-division multiplexing (MDM) in few-mode or multimode fibers that can be applied in addition to WDM and PDM. The principle of MDM in the highly multimode optical fiber (MMF) was demonstrated in the early $80^{\prime}{ }^{1}$, but due to non-desirable mode coupling MDM was feasible only over short distances. Practical implementations of MDM were delayed a few more years, until transmission systems that would support MDM met some minimal requirements which included: the development of a new fiber, optical multiplexers and demultiplexers as well as suitable amplifiers, receivers and digital signal processing techniques. In 2011 a prototype of FMF was introduced with the possibility to transmit two $100 \mathrm{~Gb} / \mathrm{s}$ PDM-QPSK data streams over two different modes along a $40 \mathrm{~km}$ optical link ${ }^{2}$. From that time the rapid progress in development MDM transmission systems begun bringing improvement in the transmission over larger distances ${ }^{3}$ and bandwidths of $\mathrm{MDM}^{4}$.

*omul@fotonik.dtu.dk; phone +45 45256632

Photonic Instrumentation Engineering II, edited by Yakov G. Soskind, Craig Olson,

Proc. of SPIE Vol. 9369, 936909 - @ 2015 SPIE · CCC code: 0277-786X/15/\$18

doi: $10.1117 / 12.2079413$

Proc. of SPIE Vol. 9369 936909-1 
With the development of MDM, FMF fibers became a key component in the transmission systems bringing a requirement of the careful fiber design with desired fiber properties like proper number of the propagating modes, low level of the cross talk between them or the minimal differential mode group delays depending on the chosen approach. One of the approaches is to minimize mode-coupling (the weakly-coupled approach) ${ }^{5}$, so each mode can be detected using a simple $2 \times 2$ or $4 \times 4$ multiple-input multiple-output (MIMO) techniques. Another one is to decrease the differential mode group delay between modes (the strongly-coupled approach), which makes possible the simultaneous detection of all modes by using complex $2 \mathrm{Nx} 2 \mathrm{~N}$ MIMO techniques. Regardless of which approach is chosen, an accurate experimental evaluation of the designed fibers is necessary.

In this contribution we demonstrate an interferometric-based approach for the FMF modal content characterization. The presented method can be used as a valuable diagnostic tool for MDM systems as an experimental technique of modal evaluation with a temporal resolution of few fs, so even the modes with a small intermodal time delay can be discovered. Estimation of the relative modal weight does not require a comparison with one of the propagating modes. The sensitivity of this method to the influence of an ambient medium is minimal and the system is stable during all measurements.

\subsection{Experimental setup}

\section{EXPERIMENT}

Figure 1 presents our experimental setup based on a Mach-Zehnder interferometer. A broadband supercontinuum laser (SuperK COMPACT, NKT Photonics) with operational wavelengths from $450 \mathrm{~nm}$ to $2400 \mathrm{~nm}$ is used as light source. To analyze the performance of the setup depending on different spectral widths, we used two bandpass filters centered at $850 \mathrm{~nm}$ with FWHM of $5 \mathrm{~nm}$ and $10 \mathrm{~nm}$ respectively. A fiber coupler is used to equally split the incoming light into reference and test arms. The test arm contains a mechanical grating unit for higher-order modes excitation ${ }^{6}$ with a fiber polarization controller, the fiber under test (FUT, SMF28 Corning, a single mode fiber with cut-off wavelength of $1260 \mathrm{~nm}, 8.2 \mu \mathrm{m}$ core diameter and $0.14 \mathrm{NA}$ ) and a linear polarizer (Thorlabs) to control the final polarization state of the output light. The reference arm consists of the single mode reference fiber (HP780, Nufern), an optical delay line and a linear polarizer. The reference and test beams recombine on a free space beam splitter producing interference fringes that are captured by a CCD camera (Point Grey, maximum frame rate 120fps) at different positions of the delay line. Information about quantity of modes in the fiber under test (FUT) and their differential time-delays could be extracted from the obtained interferograms.

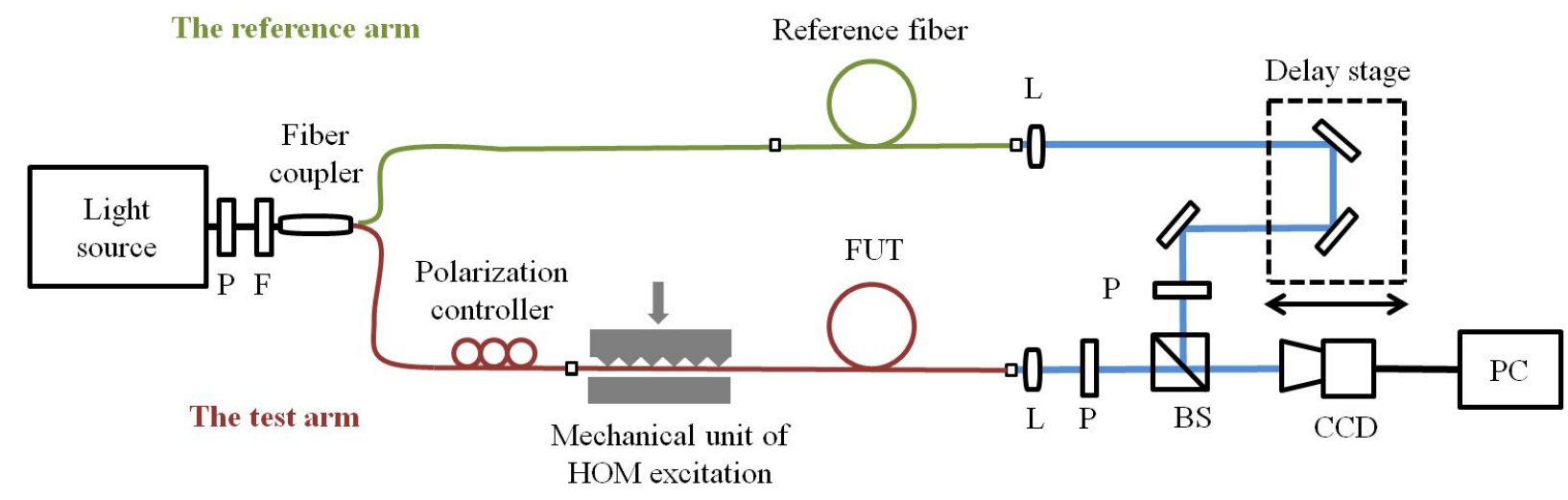

Figure 1. Experimental setup of the interferometric-based method: F - filter, L - lens, P - polarizer, BS - beam splitter, PC personal computer. Light is split into reference and test beams. Interferograms of the recombined beams are taken by CCD camera at each step of the delay line. 
For the higher-order modes excitation, the FUT is placed in the mechanical grating unit that is composed of a springloaded clamp that controls the pressure between two metallic plates: (one flat and other with $\mathrm{V}$ grooves forming a grating) as shown in Fig.1. Applying pressure to the plates creates points in the fiber where the refractive index is altered due to a photo-elastic effect. This mechanically generated periodic alteration of the refractive index possesses similar features to the photoinduced change of the refractive index in the core of a long-period grating fiber. Such periodic stressing over a beat length forces the coupling of the fundamental mode into one of the higher-order modes when a phase-matched condition is satisfied ${ }^{6,7}$.

For our experiment, a mechanical grating of period $\Lambda=532 \mu \mathrm{m}$ is chosen to covert the fundamental mode LP01 into the higher-order mode LP11 at a wavelength of $850 \mathrm{~nm}$ with a conversion efficiency of $15 \mathrm{~dB}$. In order to confirm the presence of the excited mode, the FUT is spliced with a single-mode fiber (HP780), where at 850nm only the fundamental mode can propagate. A successful mode conversion is expected to present a strong attenuation notch in the spectrum at the designed wavelength (as shown in Figure 2 (c)). The depth of the notch is relevant to the conversion efficiency that is controlled by the pressure of the spring-loaded clamp on the FUT. Higher pressure results in a stronger modal conversion. After some level of the pressure the depth of the notch does not change any more or decrease, so an optimal level of the pressure should be manually chosen. Once the perturbation stops, the light spectrum returns to its initial values (Figure 2 (b)). The inset on Figure 2 (c) represents the profile of LP11 that is obtained by directing the beam from the FUT to the camera after removing the single-mode fiber. Since this mechanical mode converter is polarization-dependent we use a polarization controller before it to optimize polarization of the coupled modes and to select LP11 only in one polarization state. Comparing to the other methods of the mode conversion this approach is simple in implementation and operation.

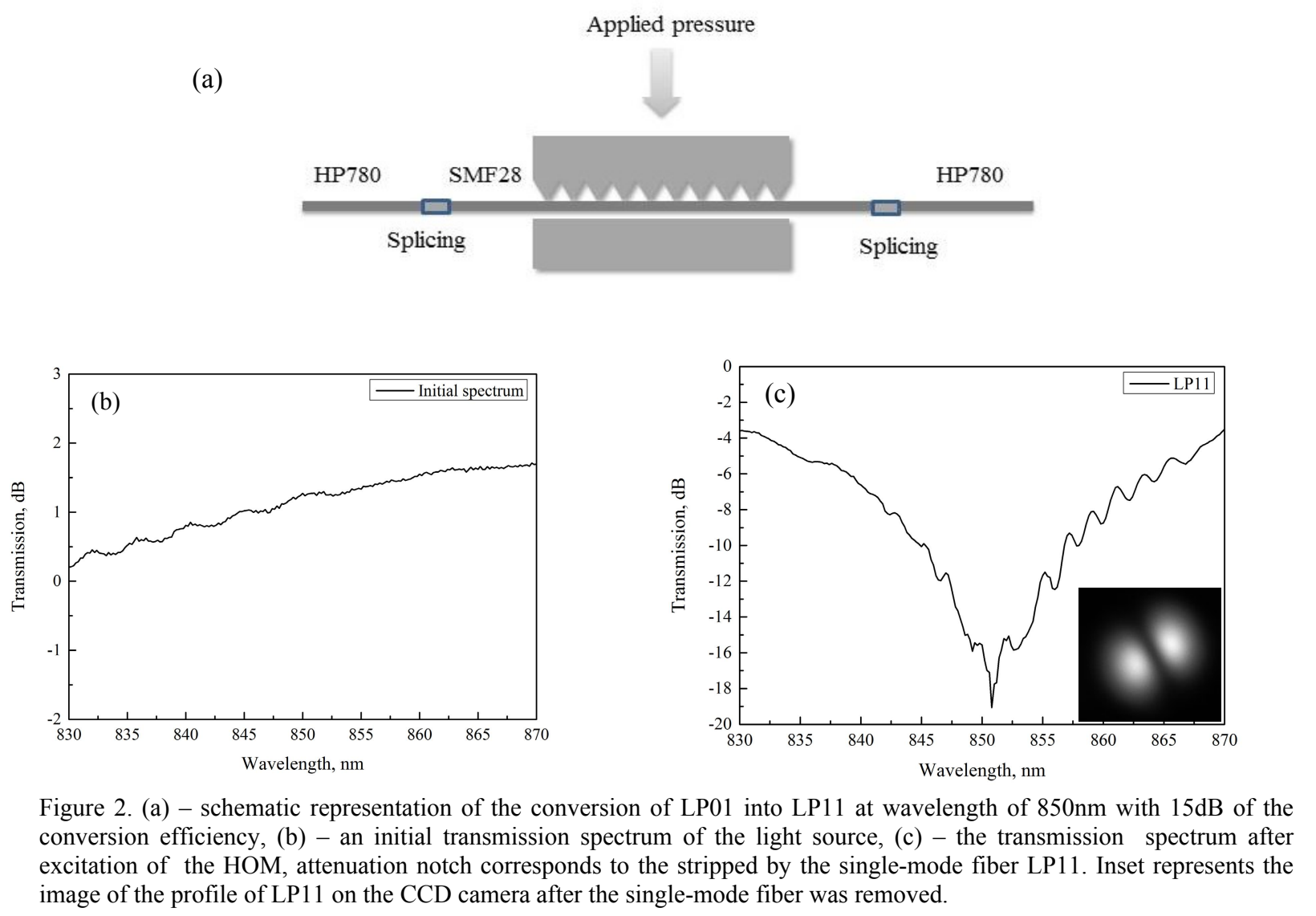




\subsection{Results}

In our experiment we are used $75 \mathrm{~cm}$ of a step-index standard fiber SMF28 (Corning) as the FUT. The active length of the fiber, from a point where the HOM is excited by the mechanical grating unit to the end facet of the fiber, is $23 \mathrm{~cm}$. At the operational wavelength of $850 \mathrm{~nm}$ in SMF28, two modes propagate: the fundamental (LP01) and the first HOM (LP11) and we therefore consider it as FMF at our operational wavelength. Simulation of the FUT proves the presence of LP01 and LP11 at 850nm with the calculated differential time-delay between modes of 1.462ps.

The reference fiber is HP780, a standard single mode fiber with a cut-off wavelength of $730 \pm 30 \mathrm{~nm}$. The optical beams from the outputs of reference fiber and the FUT recombine on the beam splitter (Figure 1). When the two arms of the Mach-Zehnder interferometer are perfectly matched, which means that the difference in their optical paths is equal or smaller than the coherence length of the light source, interference appears. An example of the interference between the excited LP11 from the FUT and the fundamental mode from the reference fiber is shown on Figure 3. The closer the path difference is to zero, the higher the contrast of the fringes on the interferograms becomes. Equation 1 shows the dependence of the coherence length with the width of the spectrum and the central wavelength. In order to obtain interferograms with a high fringe contrast when using a 10nm FWHM bandpass filter, the path difference between the two arms has to be equal or smaller than $31.79 \mu \mathrm{m}$.

$$
l_{c o h}=\frac{2 \ln (2)}{\pi} \frac{\lambda_{0}^{2}}{\Delta \lambda}=0.44 \frac{\lambda_{0}^{2}}{\Delta \lambda}
$$

where $\lambda_{0}$ is the central wavelength of the spectrum, $\Delta \lambda$ - the spectrum width.

LP01

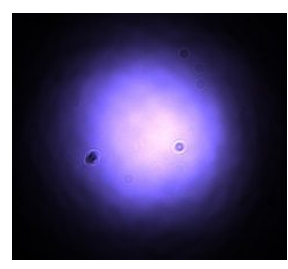

LP11

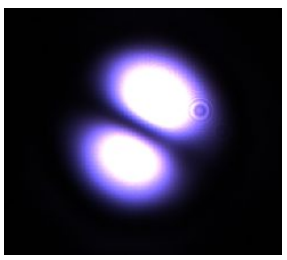

Interferogram

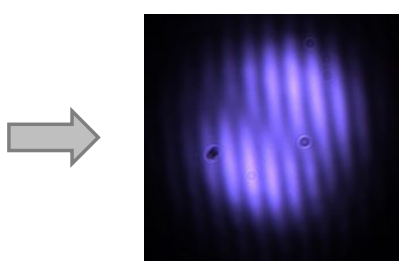

Figure 3. Interference between the LP01 from the reference fiber and excited LP11 from the FUT.

To evaluate the performance of the interferometric-based setup depending on the different coherence lengths two bandpass filters with spectral widths of $5 \mathrm{~nm}$ and $10 \mathrm{~nm}$ were used. Also we introduced visibility (V) as the parameter to assess the quality of the interference:

$$
V=\frac{I_{\max }-I_{\min }}{I_{\max }+I_{\min }}
$$

where $I_{\max }$ is the maximum brightness of the fringes on interferograms, $I_{\min }$ - the minimal brightness between the two fringes. 

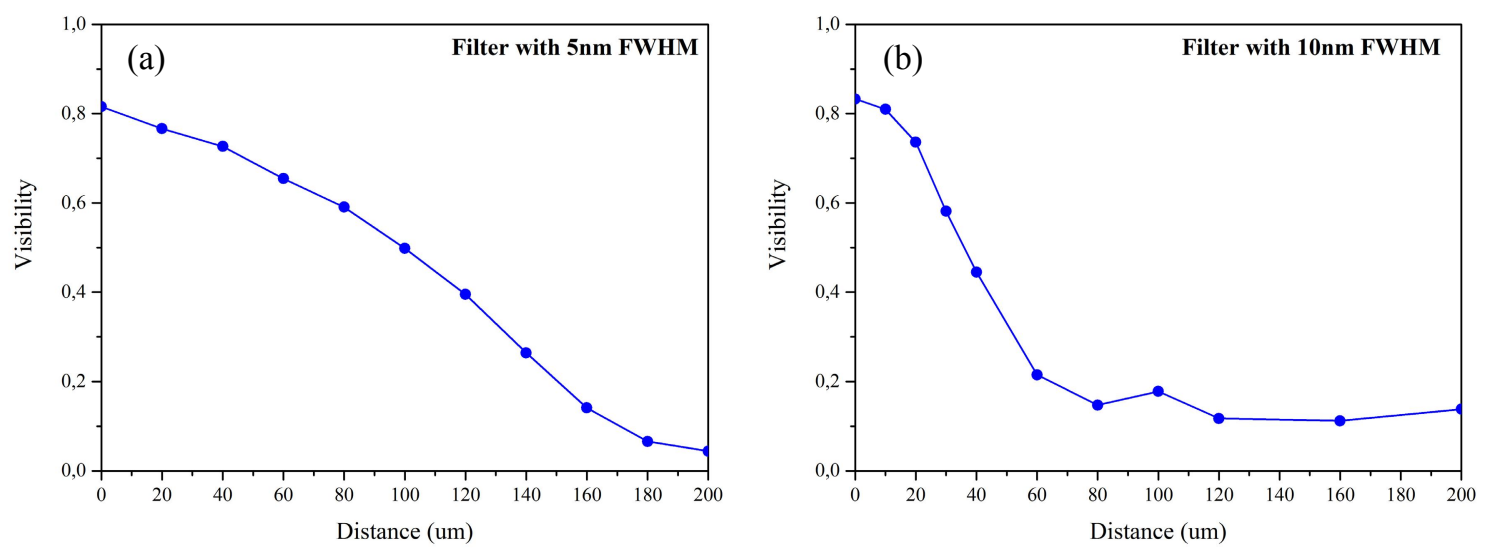

Figure 4. The visibility as a function of the optical path difference for the bandpass filters with $5 \mathrm{~nm}$ and $10 \mathrm{~nm}$ of FWHM.

At the point of the best match of the optical paths the visibility is close to 1 . The further from this point, the smaller visibility is, approaching zero after some distance. Varying the delay line we scan across the different delays of the modes in the FUT. Figures 4 (a) and (b) present the visibility as a function of the optical path difference for the bandpass filters with spectrum widths of $5 \mathrm{~nm}$ and $10 \mathrm{~nm}$ respectively. For a narrow spectrum (Figure 4) the visibility curve is broad, preserving a good contrast of the interferograms along most of the scanning length. Only one wide peak is observed. At the same time, the FWHM width of the peak is larger than the coherence length of the spectrum for $10 \mathrm{~nm}$ filter that is equal to $63.58 \mu \mathrm{m}$ due to dispersion broadening.

For a wider spectrum (Figure 4 (b)) the coherence length is equal to $31.79 \mu \mathrm{m}$, which is also smaller than the FWHM width of the peak. We suppose that a small peak at the distance of $100 \mu \mathrm{m}$ is present due to steep edges of the filtered spectrum. Comparing results for those two measurements it is evident that the temporal resolution of the system directly depends on the spectral width of the light source (as expected). The wider the initial spectrum is, the higher the temporal resolution becomes. Therefore, in order to reach a high temporal resolution in the system a light source of low coherence is required.
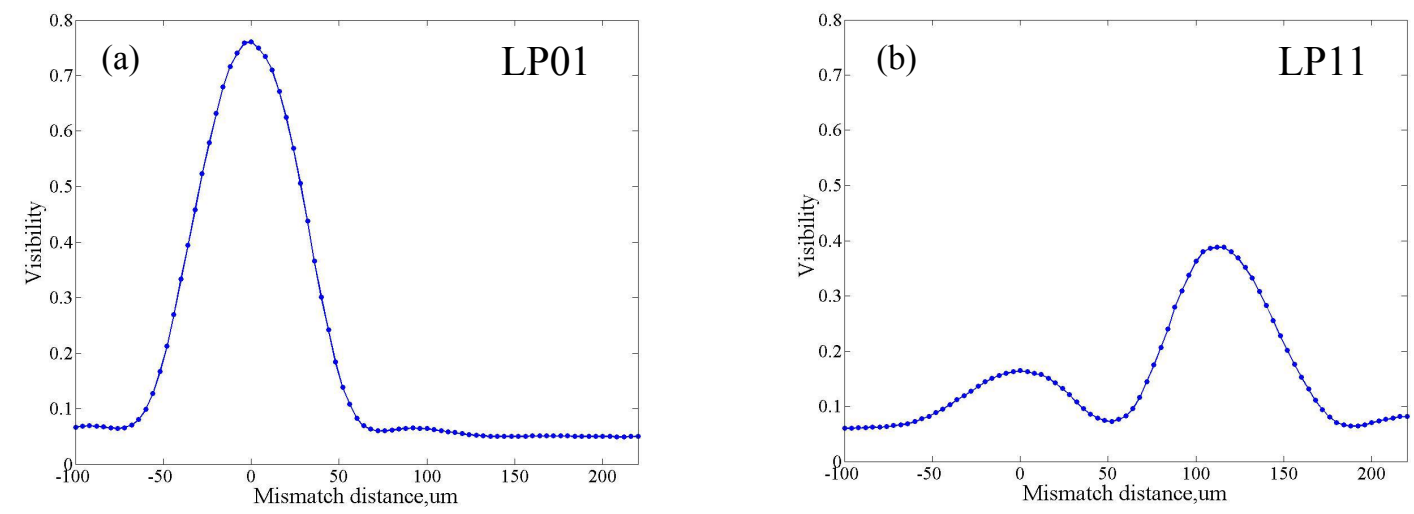

Figure 5. Experimental results of the measurement of the visibility as a function of the optical path difference for the bandpass filter with 10nm of FWHM. (a) - Only the fundamental LP01 is propagating, (b) - LP01 is partly converted into LP11.

Figure 5 depicts the experimental results from the measurements of the visibility for (a) LP01 and (b) both LP11 and LP01. The FUT was scanned along $300 \mu \mathrm{m}$ with a step of $4 \mu \mathrm{m}$. The point where interference for LP01 is higher (Figure 
5(a)) corresponds to an optical path difference of zero (origin of the visibility curve). Figure 5(b) presents a second peak, shifted $115 \mu \mathrm{m}$ from zero that corresponds to the center of the peak due to of LP11 excitation. To verify the performance of the setup LP11 was deliberately excited in order to have two modes simultaneously propagating in the fiber. It is worth noticing that this technique allows for the determination of the different modes even when the fundamental is not dominant, as in Figure 5 (b) where height of the peak of the LP01 mode is about half of that on the corresponding LP11 peak. The visibility of LP11 is 0.4 while the visibility of LP01 is 0.76 . The measured differential time-delay between LP01 and LP11 is equal to 0.383 ps.

\section{CONCLUSION}

In the current work we have presented an efficient interferometric-based technique that is a simple and practical characterization tool for the evaluation of the modal content of an optical fiber. We showed that this method allows recovering the propagating modes in FMF without prior knowledge of the fiber properties during measurement, even when the fundamental is not the dominant mode. The first HOM (LP11) was successfully excited with the conversion efficiency of $15 \mathrm{~dB}$ in the standard SMF28 fiber, which at the operational wavelength of $850 \mathrm{~nm}$ behaves as FMF. By analyzing the visibility trace an accurate estimation of the propagating modes and their relative differential time-delay is achieved. As it was shown above, current technique can become a valuable characterization tool for MDM systems.

\section{ACKNOWLEDGMENTS}

The authors gratefully acknowledge funding support from Innovationsfonden E-SPACE project \#0603-00514B

\section{REFERENCES}

[1] Berdague, S.., Facq, P., "Mode division multiplexing in optical fibers," Appl. Opt. 21(3), 1950-1599 (1982).

[2] Salsi, M., Koebele, C., Sperti, D., Tran, P., Brindel, P., Mardoyan, H., Bigo, S., Boutin, A., Verluise, F., et al., "Transmission at 2x100Gb/s, over Two Modes of 40km-long Prototype Few-Mode Fiber, using LCOS based Mode Multiplexer and Demultiplexer," Opt. Fiber Commun. Conf. Fiber Opt. Eng. Conf. 2011, PDPB9, Osa, Washington, D.C. (2011).

[3] Randel, S., Ryf, R., Gnauck, A. H., Mestre, M. A., Schmidt, C., Essiambre, R., Winzer, P. J., Delbue, R., Pupalaikis, P., et al., "Mode-Multiplexed $6 \times 20$-GBd QPSK Transmission over 1200-km DGD-Compensated Few-Mode Fiber," Opt. Fiber Commun. Conf. Expo. (OFC/NFOEC), 2012 Natl. Fiber Opt. Eng. Conf.(I), 3-5 (2000).

[4] Mori, T., Sakamoto, T., Wada, M., Yamamoto, T.., Yamamoto, F., "Few-Mode Fibers Supporting More Than Two LP Modes For Mode-Division-Multiplexed Transmission With MIMO DSP,” J. Light. Technol. 32(14), 2468-2479 (2014).

[5] Sillard, P., Bigot-Astruc, M.., Molin, D., "Few-Mode Fibers for Mode-Division-Multiplexed Systems,” J. Light. Technol. 32(16), 2824-2829 (2014).

[6] Savin, S., Digonnet, M. J. F., Kino, G. S.., Shaw, H. J., "Tunable mechanically induced long-period fiber gratings," Opt. Lett. 25(10), 710 (2000).

[7] Youngquist, R. C., Brooks, J. L.., Shaw, H. J., “Two-mode fiber modal coupler,” Opt. Lett. 9(5), 177 (1984). 